\title{
Comparison of Pain on Injection of Propofol MCT with Propofol LCT
}

\author{
Sanjot Sudhir Ninave ${ }^{1}$, Bhavitha Venigalla ${ }^{2}$ \\ ${ }^{1}$ Department of Anaesthesiology, Datta Meghe Institute of Medical Sciences, Jawaharlal Nehru \\ Medical College, Sawangi, Maharashtra, India. ${ }^{2}$ Department of Anaesthesiology, Datta Meghe \\ Institute of Medical Sciences, Jawaharlal Nehru Medical College, Sawangi, Maharashtra, India.
}

\section{ABSTRACT}

\section{BACKGROUND}

Propofol is one of the safest drugs for induction of general anaesthesia. Routinely available preparation in the market being propofol LCT (long chain triglycerides) has the disadvantage of pain on injection. This pain is very discomforting for the patient and also to the anaesthesiologist. So, they are coming up with a new preparation propofol MCT (medium chain triglycerides) which causes less pain on injection. We wanted to compare the occurrence of pain on injection of Propofol MCT with Propofol LCT.

\section{METHODS}

After obtaining institutional ethical committee approval, 150 patients undergoing various elective surgeries under general anaesthesia where propofol was used as an induction agent, aged between 18 and 65 years of ASA I \& II were enrolled in the study. The study patients were divided into two groups. Group M, who received Propofol MCT and group L, who received Propofol LCT as an induction drug. The intensity of pain was evaluated by using Visual Analogue Score (VAS). Similarly change of triglyceride levels after single induction dose of Propofol MCT and Propofol LCT were observed.

\section{RESULTS}

Group M showed reduced pain score (4.15 \pm 1.90$)$ after injection along with less incidence of pain compared to Group L (6.37 \pm 2.49 ). Serum triglyceride levels had no significant difference in preoperative and postoperative values.

\section{CONCLUSIONS}

Pain on injection with Propofol MCT $(4.15 \pm 1.90)$ is less compared to Propofol LCT $(6.37 \pm 2.49) \&$ no evidence of change of triglyceride levels was seen after a single induction dose in the study.

\section{KEY WORDS}

Propofol, Pain on Injection, Propofol MCT, Propofol LCT, Serum Triglycerides
Corresponding Author: Dr. Bhavitha Venigalla, Flat No. 9, Meghe Heights-3, Datta Meghe Institute of Medical Sciences, Sawangi, Meghe-442001, Maharashtra, India.

E-mail: bhavitha123@gmail.com

DOI: $10.14260 /$ jemds/2020/232

Financial or Other Competing Interests: None.

How to Cite This Article:

Ninave SS, Venigalla B. Comparsion of pain on injection of propofol MCT with propofol LCT. J. Evolution Med. Dent. Sci. 2020;9(13): 1078-1081, DOI: 10.14260/jemds/2020/232

Submission 29-01-2020, Peer Review 11-03-2020, Acceptance 18-03-2020, Published 30-03-2020.

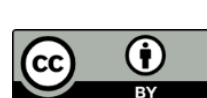




\section{BACKGROUND}

Pain is defined by International Association for the Study of Pain (IASP) as "an unpleasant sensory and emotional experience associated with actual or potential tissue damage or described in terms of such damage.(1) Propofol is a substituted Isopropyl phenol that is administered intravenously as $1 \%$ solution in an aqueous solution of $10 \%$ soya bean oil, $2.25 \%$ glycerol and $1.2 \%$ purified egg phosphatide. The mechanism of pain on injection of propofol is thought to be multifactorial but its exact causation is not clear. The most commonly identified mechanism is release of bradykinin as a result of the activation of the plasma kininkallikrein system by propofol.(2) Aqueous phase of free propofol is considered to be the cause of injection pain of propofol.(3) Propofol is currently the preferred intravenous general anaesthetic drug with a smooth induction, pleasant sleep, rapid recovery, and low incidence of nausea and vomiting. Despite these positive properties, it also has adverse effects such as injection pain, which causes discomfort in the induction of anaesthesia.(4) Patients have reduced satisfaction due to this unpleasant sensation of pain.

Attenuation of this pain is commonly done using on intravenous lidocaine, addition of lidocaine to propofol results in coalescence of oil droplets,(5),(6) thought that the concentration of free propofol in the aqueous phase can be changed. Different drugs available for attenuating pain on injection apart from lignocaine are ketamine, opioids, nonsteroid anti-inflammatory drugs, inhalational agents such as nitrous oxide $\left(\mathrm{N}_{2} \mathrm{O}\right)$ and sevoflurane, clonidine, dexamethasone, and others.(7) Lignocaine role in attenuating injection pain associated with propofol is a norm almost for many years, there are many clinical trials alone or in combination with other drugs given following venous occlusion using tourniquet up to 45-60 seconds. Effective dose being $60 \mathrm{mg}$, whereas $40 \mathrm{mg}$ is most commonly used.(8) Ketamine is also considered for injection pain of propofol, but has undesirable adverse effects of secretion production and hemodynamic responses.(9) Instead of using different drugs with or without venous occlusion., there is need of propofol solution which by itself is painless, so we want to compare Propofol MCT with that of routinely used Propofol LCT. The pharmaco-kinetics and propofol efficacy does not have any effect on the composition of carrier fat emulsion.(10) However with comparison to other studies the acceptance of propofol MCT/LCT by patient because it decreases the severity and incidence of severe to moderate pain.

The results of this study showing that the concentration of free propofol is significantly smaller in propofol MCT/LCT than in propofol LCT are consistent with previously reported suggestions that the use of propofol MCT/LCT reduces the incidence and intensity of pain on injection.(10),(11),(12),(13) Adding to the additional note regarding potential risks related to propofol however, an increase in serum triglyceride levels has been described repeatedly, particularly after long-lasting infusions. In critically ill patients presenting with deranged metabolic or enzymatic systems, prolonged propofol administration might result in an excessive fat load with ensuing pancreatitis, which is a well-known complication of hyper triglyceridemia.(14) So we considered to compare the pain on injection and if the single bolus dose of propofol do elevate serum triglycerides levels or not.
We wanted to study the efficacy of Propofol MCT over routinely use Propofol LCT in attenuating the pain on injection $\&$ any change of serum triglyceride levels after single bolus induction dose.

\section{METHODS}

This Prospective comparative study was carried out in the Department of Anaesthesiology, JNMC, Sawangi (Meghe), Wardha, after approval from the Institutional Ethics Committee. Assuming VAS score of Propofol MCT 2.089 and SD of 0.896 , keeping power at $80 \%$ and confidence interval at $95 \%$ (alpha error at 0.05 ) a sample of 60 patients would be required to detect a minimum of $25 \%$ of pain on IV Propofol MCT and LCT. We include 75 patients in each group to compensate for possible drop out. A total of 150 patients aged between 18 - 65 years willing to give written informed consent fitting into the inclusion criteria were included in this prospective comparative study scheduled for various elective surgeries under general anaesthesia. Patients are divided into groups into 75 each. Group MCT received 25\% of Propofol MCT induction dose. Group LCT received $25 \%$ of propofol LCT. Pre anaesthetic evaluation was done a day before the surgery. Patients were asked to be nil by mouth by 8 hours. All the necessary routine investigations were noted along with preoperative triglycerides. Patients with chronic pain disorder, known allergy to the study drug, pregnancy, abnormal renal and liver function were excluded from the study. On arrival of patient to operation theatre, all routine monitors are attached to the patient and baseline parameters such as HR, SBP, DBP, MAP, SPO2 were recorded. Intra-venous cannulation was done using wide bore cannula (18 G or $20 \mathrm{G}$ ) on the dorsum of the hand or forearm. Pre-medications were deliberately avoided to avoid influence on study results. With a tourniquet in place distal to venous cannulation. $2.5 \mathrm{ml}$ of total $10 \mathrm{ml}$ propofol i.e. $25 \%$ of the induction dose is given to according to the groups divided. Patients are asked to indicate the severity of pain on injection using VAS Score,(15) at 25 seconds. This is the end point of the study and further procedure was carried out in conventional manner depending on the type of surgery. Awareness of the pain due to propofol after general anaesthesia is not considered. Postoperative serum triglyceride levels were measured.

\section{Statistical Analysis}

Descriptive and analytical statistics were done. The normality of continuous data was analysed by the Shapiro-Wilk test. As the data followed normal distribution, parametric test was used to analyse the data. The independent sample t-test and paired sample t-test were used to check mean differences wherever appropriate. The chi-square test was used to check differences in proportions. The level of significance was kept at $\mathrm{p}<0.05$.

\section{RESULTS}

In all the patients demographic details in table 1 , such as age, sex, weight and ASA status were comparable between both groups .The VAS pain score is compared between Group L 6.37 
\pm 2.49 (Propofol LCT) and Group M $4.15 \pm 1.90$ (Propofol MCT),Group M reported significantly reduced pain after IV propofol injection $(p<0.001)$ compared to Group L. There is an overall less incidence of pain in Group M (54\%) compared to Group L (65\%). Induction time in seconds when compared between Group L $(34.07 \pm 2.68)$ to Group M $(33.61 \pm 2.72)$ was similar, and had no difference. When comparison of serum triglycerides was done preoperatively and postoperatively showed no difference.

\begin{tabular}{|cccc|}
\hline MAP & N & Group L & Group M \\
Age (Mean \pm SD) & 75 & $44.31 \pm 11.29$ & $41.85 \pm 10.19$ \\
Gender & & & \\
Male [n (\%)] & 75 & $40(53.3)$ & $41(54.7)$ \\
Female [n (\%)] & & $35(46.7)$ & $34(45.3)$ \\
Weight (Mean \pm SD) & 75 & $59.67 \pm 9.53$ & $61.92 \pm 12.51$ \\
ASA Grade & 75 & $57(76.0)$ & $59(78.7)$ \\
ASA I [n (\%)] & 75 & $18(24.0)$ & $16(22.3)$ \\
ASA II [n (\%)] & Table 1. Demographic Data of the Two Groups - Propofol LCT \& \\
\hline & Propofol MCT \\
\hline
\end{tabular}

\begin{tabular}{|c|c|c|c|c|c|}
\hline MAP & $\mathbf{N}$ & Group L & Group M & P-Value & Sig. \\
\hline VAS Pain Score (Mean \pm SD) & 75 & $6.37 \pm 2.49$ & $4.15 \pm 1.90$ & $<0.001$ & $\mathrm{~s}$ \\
\hline $\begin{array}{l}\text { Induction time in seconds } \\
(\text { Mean } \pm \text { SD })\end{array}$ & 75 & $34.07 \pm 2.68$ & $33.61 \pm 2.72$ & 0.306 & NS \\
\hline Pain Present [n (\%)] & 75 & $65(86.7)$ & $54(72.0)$ & 0.027 & $\mathrm{~S}$ \\
\hline $\begin{array}{l}\text { Table 2. Comparison o } \\
\text { Presence of Pain betwe }\end{array}$ & $\begin{array}{l}\text { Yea } \\
\text { the }\end{array}$ & $\begin{array}{l}\text { VAS Pain Sc } \\
\text { Two Groups } \\
\text { MCT }\end{array}$ & $\begin{array}{l}\text { core, Induct } \\
\text { - Propofol L }\end{array}$ & $\begin{array}{l}\text { tion Time } \\
\text { LCT \& Pro }\end{array}$ & $\begin{array}{l}\text { ind } \\
\text { oofol }\end{array}$ \\
\hline
\end{tabular}

\begin{tabular}{|c|c|c|c|}
\hline Triglycerides & $\mathbf{N}$ & $\begin{array}{l}\text { Group L } \\
\text { Mean } \pm \text { S.D. }\end{array}$ & $\begin{array}{c}\text { Group M } \\
\text { Mean } \pm \text { S.D. }\end{array}$ \\
\hline Pre-Op Triglycerides & 75 & $128.94 \pm 21.62$ & $128.62 \pm 21.59$ \\
\hline Post-Op Triglycerides & 75 & $134.41 \pm 20.44$ & $132.44 \pm 12.76$ \\
\hline P-Value & & 0.099 & 0.174 \\
\hline Significance & & NS & NS \\
\hline \multicolumn{4}{|c|}{$\begin{array}{l}\text { Table 3. Comparison of Pre and Post Triglycerides Values } \\
\text { of Group Propofol LCT and Propofol MCT }\end{array}$} \\
\hline
\end{tabular}

\section{DISCUSSION}

Propofol is most commonly used inducing agent in the practice of anaesthesia providing smooth induction, rapid recovery and low incidence of nausea and vomiting.

Propofol (2,6-diisopropylphenol) and lipophilic weak acid $\left(\mathrm{p} K_{\mathrm{a}}=11\right)$ is extremely insoluble in water, there is a necessary to formulate it in a lipid-based emulsion, Containing soya bean oil, glycerol, and egg lecithin. As it can be conducive to bacterial growth, addition of the chelating agent disodium edetate has reduced this (16) . Propofol has a better safety profile. Few of the most common side effects being dose-dependent hypotension and cardiorespiratory depression. Propofol being a global central nervous system (CNS) depressant. It activates $\gamma$-aminobutyric acid (GABA A) receptors directly, inhibits the $\mathrm{N}$-methyl-D-aspartate (NMDA) receptor and modulates calcium influx through slow calcium-ion channels. Propofol has a anxiolytic effect at doses that do not produce sedation, mechanism of its anxiolytic action is mostly due to to a positive modulation of the inhibitory function of GABA through GABAA receptors. Apart from anxiolytic action ,it also has immunomodulatory activity for diminishing the systemic inflammatory response. It has also been reported that, propofol has neuroprotective effects, decreases the cerebral blood flow and decreases intracranial pressure (ICP). Propofol is also a potent antioxidant and has anti inflammatory properties(17).
Phenols have the tendency to irritate skin and mucous membrane, Propofol (alkylphenol )is expected to cause pain on injection in spite of being isotonic. This pain is described as angialgia [18] meaning pain is due to vascular involvement (18).site of injection, size of vein, speed of injection, propofol concentration in aqueous phase, buffering effect of blood are some of the factors which appeared to affect incidence of pain of propofol injection(19) Despite it's positive effects ,pain on propofol injection is ranked $7^{\text {th }}$ amongst the most important 33-low morbidity clinical anaesthesia problems by a panel of expert anaesthesiologists (20). The Pain of propofol injection may be severe enough to cause life-threatening complications like triggering severe bronchospasm in a smoker(21) and myocardial ischemia attack due to profound pain(22). Options available for attenuating pain on injection with propofol include use of local anaesthetic (lignocaine, most widely used) ketamine, opioids, non-steroid anti-inflammatory drugs, inhalational agents such as nitrous oxide $\left(\mathrm{N}_{2} \mathrm{O}\right)$ and sevoflurane, clonidine, dexamethasone, and others(7)Pain caused during propofol injection can be attenuated by formulation in medium chain, rather than long-chain triglycerides(16).

In our study we compare the efficacy of Propofol MCT with Propofol LCT.Table 1 shows Patient characterstics pertaining to demographic details and ASA grade, did not differ between both the groups. Table 2 shows The VAS score in Propofol LCT $(6.37 \pm 2.49)$ is more compared to Propofol MCT $(4.15 \pm 1.90)$. Similarly, Rau et al reported less injection pain with Propofol MCT/LCT ( $p=0.0007)$ (13). Table 2 shows that incidence of pain in more in Propofol LCT (86.7 \%) compared to Propofol MCT (72.0\%). Larsen et al reported Propofol MCT/LCT had significantly lower incidence of pain on injection in comparison with standard propofol group LCT (37\% vs $65 \%$ ). (12) Table 2 compared the Induction time in Group L (34.07 \pm 2.68$)$ and Group M (33.61 \pm 2.72$)$, was found to be similar. Table 3 Compared the pre-operative and postoperative triglycerides which showed no significant difference between both the groups, similar to Manjula et al failed to increase triglyceride levels to a significant level, despite the difference in the lipid content, single dose of MCT/LCT or LCT propofol did not increase serum triglyceride levels significantly to cause any adverse effects(23) but Bhukal et al demonstrated that both LCT and MCT-LCT propofol cause significant rise in triglyceride levels in children when used for induction and maintenance of anaesthesia. However, children in MCT-LCT group had lower triglyceride levels than children in LCT group at the end of propofol infusion and 4 hours after termination.(24)

Adding on to details Ali et al stated that increased serum triglyceride level after propofol infusion is associated with increased risk of pancreatitis, coronary artery disease. It occurs in ICU patients who receive long term propofol infusion ( $>24$ hrs.).(25) But this propofol infusions are not now routinely used as newer and better drugs like dexmedetomidine and others have taken over propofol. No studies have cited that there was increase in serum triglycerides after single bolus dose of propofol except a case of 21-year-old patient operated for Bartholin duct excision developing pancreatitis after single dose of propofol.(26)

Various options were tried for the prevention of injection pain caused by propofol with varying degree of success one amogst it is use of lidocaine. There is sole need of propofol 
solution which by itself is painless or less painful. The draw back of propfol being pain on injection may be distressing to patient, interfere with smooth induction, which can be attenuated by a formulation in médium chain triglycerides rather than long chain triglycerides similar to Rau et al.(13)

\section{CONCLUSIONS}

Propofol MCT was associated with less incidence of pain on injection, compared to Propofol LCT, and does not need addition of any other drug to reduce this pain of injection. Also, the formulation did not increase serum triglyceride levels after single bolus dose.

\section{REFERENCES}

[1] Watson J. PAIN MECHANISMS - A REVIEW: I. Characteristics of the peripheral receptors. Aust J Physiother [Internet]. 1981;27(5):135-43. Available from: http://dx.doi.org/10.1016/S0004-9514(14)60753-4

[2] Haydar B. Stoelting's Pharmacology and Physiology in Anesthetic Practice, 5th Edition: Stoelting's Handbook of Pharmacology and Physiology in Anesthetic Practice, 3rd Edition. Anesthesiol J Am Soc Anesthesiol [Internet]. 2015 Jun 1;122(6):1445. Available from: https://doi.org/10.1097/ALN.0000000000000646

[3] Mangar D, Holak EJ. Tourniquet at $50 \mathrm{~mm} \mathrm{Hg}$ Followed by Intravenous Lidocaine Diminishes Hand Pain Associated With Propofol Injection. Anesth Analg [Internet]. 1992;74(2). Available from: https://journals.lww.com/anesthesiaanalgesia/Fulltext/1992/02000/Tourniquet_at_50_mm_H g_Followed_by_Intravenous.14.aspx

[4] Beyaz S, Eman A. Injection pain of propofol in children: A comparison of two formulations without added lidocaine. J Anaesthesiol Clin Pharmacol. 2012;28(3):314-7.

[5] Kennedy A. The effect of the addition of lignocaine on propofol emulsion stability. 2006;5(January 1996):18-21.

[6] Park JW, Park E, Lee K. The Effect of Lidocaine on the Globule Size Distribution of. 2003;769-71.

[7] Desousa KA. Pain on propofol injection: Causes and remedies. Indian J Pharmacol [Internet]. 2016;48(6):61723. Available from: https://www.ncbi.nlm.nih.gov/pubmed/28066096

[8] Kim DH, Chae YJ, Chang HS, Kim JA, Joe HB. Intravenous lidocaine pretreatment with venous occlusion for reducing microemulsion propofol induced pain: comparison of three doses of lidocaine. J Int Med Res [Internet]. 2014/03/04. 2014 Apr;42(2):368-75. Available from: https://www.ncbi.nlm.nih.gov/pubmed/24595146

[9] Iwata $M$, Inoue $S$, Kawaguchi $M$, Kimura $T$, Tojo $T$, Taniguchi S, et al. Ketamine eliminates propofol pain but does not affect hemodynamics during induction with double-lumen tubes. J Anesth [Internet]. 2010;24(1):31-7. Available from: https://doi.org/10.1007/s00540-0090833-5

[10] Connor O, Doenicke AW, Kugler J, Klotz U, Roizen F, Rau J. Pharmacokinetics a New Solvent and Pharmacodynamics of Propofol. 1997;
[11] Müller RH, Harnisch S. Physicochemical characterization of propofol-loaded emulsions and interaction with plasma proteins. Eur Hosp Pharm. 2000 Jan 1;6.

[12] Larsen B, Beerhalter U, Biedler A, Brandt A, Doege F, Brün $\mathrm{K}$, et al. [Less pain on injection by a new formulation of propofol? A comparison with propofol LCT]. Anaesthesist [Internet]. 2001 Nov;50(11):842-845. Available from: https://doi.org/10.1007/s00101-001-0234-0

[13] Rau J, Roizen MF, Strohschneider U, Doenicke AW, Connor MFO. Propofol in an Emulsion of Long- and Medium-Chain Triglycerides: The Effect on Pain. 2001;(Table 1):382-4.

[14] Theilen HJ, Adam S, Ragaller M, Albrecht MD. Propofol in a Medium- and Long-Chain Triglyceride Emulsion: Pharmacological Characteristics and Potential Beneficial Effects. 2002;

[15] Correll D. The Measurement of Pain: Objectifying the Subjective. Pain Manag. 2006 Dec 1;1:197-211.

[16] Hayes I, Fjficmanz F. Pharmacology of anaesthetic agents I : intravenous anaesthetic agents. 2014;14(3):100-5.

[17] Kotani Y, Shimazawa M, Yoshimura S, Iwama T, Hara H. The experimental and clinical pharmacology of propofol, an anesthetic agent with neuroprotective properties. CNS Neurosci Ther. 2008;14(2):95-106.

[18] Rasmussen KG, Ritter MJ. Anesthetic-induced pain on injection in electroconvulsive therapy: review of the literature and suggestions for prevention. J ECT. 2014 Sep;30(3):203-9.

[19] Huang YW, Buerkle H, Lee TH, Lu CY, Lin CR, Lin SH, et al. Effect of pretreatment with ketorolac on propofol injection pain. Acta Anaesthesiol Scand. 2002 Sep;46(8):1021-4.

[20] Macario A, Weinger M. Which Clinical Anesthesia Outcomes Are Both Common and Important to Avoid? The Perspective of a Panel of Expert Anesthesiologists. 1999;

[21] Zeidan A. Profound pain due to propofol injection triggered severe bronchospasm in a smoker--A case report. Middle East J Anaesthesiol. 2006 Jun;18(5):93942.

[22] Profound Pain Due to Propofol Injection Triggered Myocardial Ischemia in a Patient with a Suspected Pheochromocytoma Rapacuronium: Premarket Drug Evaluation Can Be Very Effective for the Identification of Drug Risks. 2003;2003.

[23] Sarkar MS, Desai PM, Sageer H, Sarkar S. Propofol LCT vs propofol MCT-LCT: Randomized controlled trial. 2016;3(2):214-8.

[24] Bhukal I. Comparison of serum triglyceride levels with propofol in long chain triglyceride and propofol in medium and long chain triglyceride after short term anesthesia in pediatric patients. 2014;8(November).

[25] Devlin JW, Lau AK, Tanios MA. Propofol-associated hypertriglyceridemia and pancreatitis in the intensive care unit: an analysis of frequency and risk factors. Pharmacotherapy [Internet]. 2005 Oct;25(10):1348-52. Available from: https://www.ncbi.nlm.nih.gov/pubmed/16185179

[26] Jawaid Q, Presti ME, Neuschwander-Tetri BA, Burton FR. Acute pancreatitis after single-dose exposure to propofol: a case report and review of literature. Dig Dis Sci [Internet]. 2002 Mar;47(3):614-8. Available from: https://www.ncbi.nlm.nih.gov/pubmed/11911351 